\title{
Phase Diagrams for Three Component Mixtures in Pharmaceuticals and its Applications
}

\author{
Abhishek Sunil Dhoot, Anup Naha*, Juhi Priya, Neha Xalxo \\ Department of Pharmaceutics, Manipal College of Pharmaceutical Sciences, Manipal Academy of Higher Education, Manipal, Karnataka, INDIA.
}

\begin{abstract}
A phase is a physically discrete and chemically homogeneous portion of a system, separated by boundaries from other portions of the system. The phase rule given by J.W Gibbs in 1874 is applied to define a system composed of single component as well as multi-component. Various pharmaceutical formulations such as emulsion, micro emulsions and gels are mainly composed of lipid soluble component, water soluble component, surfactant and cosolvents. Phase diagram serves as an important tool for screening of such formulation components. A phase diagram is a graphical representation composed of area curve, lines and points, which is used to describe a specific system composed of a single or multiple components and generate the data with respect to concentration of different components, temperature and pressure at which thermodynamically distinct phases occur, transform from one phase to another and coexist at equilibrium. A ternary phase diagram has the shape of a triangular prism with an equilateral triangle as a foundation which is used to describe a three component system. Ternary phase diagrams give the precise and accurate data of the
\end{abstract}

various composite component systems and are utilized in several areas of pharmaceuticals like the formulation of various dosage forms, polymer coating methods and several other formulation processes. The review focuses on the concept of phase diagram and phase rule, various methods and rules relating to ternary phase diagrams, various examples and its recent applications in pharmaceutical research.

Key words: Phase rule, Phase diagrams, Ternary phase diagram, Three component system, Fish diagram.

Correspondence

Dr. Anup Naha, Department of Pharmaceutics, Manipal College of Pharmaceutical Sciences, Manipal Academy of Higher Education, Manipal, Karnataka, INDIA.

Phone: +91-9739314682

Email: anup.naha@manipal.edu

DOI: 10.5530/jyp.2018.10.31

\section{INTRODUCTION}

A phase can be described as chemically homogeneous and physically discrete part or portion of a system that is separated from other parts or portions of a system by boundaries. All the parts of a system can be termed as components. To define a particular system or state of each phase in a system, a fixed number of variables have to known. The phase rule was given by J.W Gibbs in 1874. The phase rule is based on thermodynamics and helps to determine the condition for non-reactive multi-component heterogeneous or polyphase system to exhibit phase equilibrium. ${ }^{1-6}$

Phase rule can be defined as 'When a heterogeneous system in equilibrium at a definite temperature and pressure, the number of degrees of freedom is equal to by 2 the difference in the number of components and the number of phases provided the equilibrium is not influenced by external factors such as gravity, electrical or magnetic forces, surface tension etc. ${ }^{2}$

Mathematically, it can be written as $\mathbf{F}=\mathbf{C}-\mathbf{P}+\mathbf{2}$

Here, the $\mathrm{F}$ is the number of degrees of freedom in system or the minimum numbers of factors that have to be known to define a system, $\mathrm{C}$ is the number of independent components or parts of a system and $\mathrm{P}$ is the number of phases present in the system. ${ }^{3}$

The phase rule is applied to define a system composed of single component as well as multi-component. Phase diagrams can be defined as graphical representation consist of area curve, lines and points which are used to show several conditions such as temperature and pressure at which thermodynamically distinct phases occur, transform from one phase to another and coexist at equilibrium. ${ }^{3}$

The system can be called as in-variant, uni-variant, bi-variant and so on as per to degrees of freedom (F) is zero, one, two and so on. For example, in a three component system with two phases, degree of freedom will be one or the system is will be univariant. ${ }^{4}$

For one component system such as water, the phase rule is enforced and the phase diagram gives a batch of information like the sublimation temperature, triple point, boiling and freezing temperature. As per phase rule equation, a one component system should have maximum two degrees of freedom. For two component systems like Water and phenol, which are partially miscible, the phase diagram gives the information regarding the various concentrations at which the two phases are miscible with each other and the temperature at which they are miscible. A two component system consisting Salol-thymol system shows four regions in the phase diagram, information such as the melting point of Individual components can be established. A phase diagram is used to establish the 'eutectic point' at which the solid and liquid phases exhibit the same composition. It is very important because the eutectic point is at a temperature lower than the melting point of either of the individual components. Thus, a eutectic mixture can melt at a temperature lower than the temperature required to melt either of the individual components. Ternary phase diagrams give the precise and accurate data of the various composite component systems and are utilized in several areas of pharmaceuticals like the formulation of emulsions, micro-capsules, gels, polymer coating methods and several other formulation processes. ${ }^{3}$ 


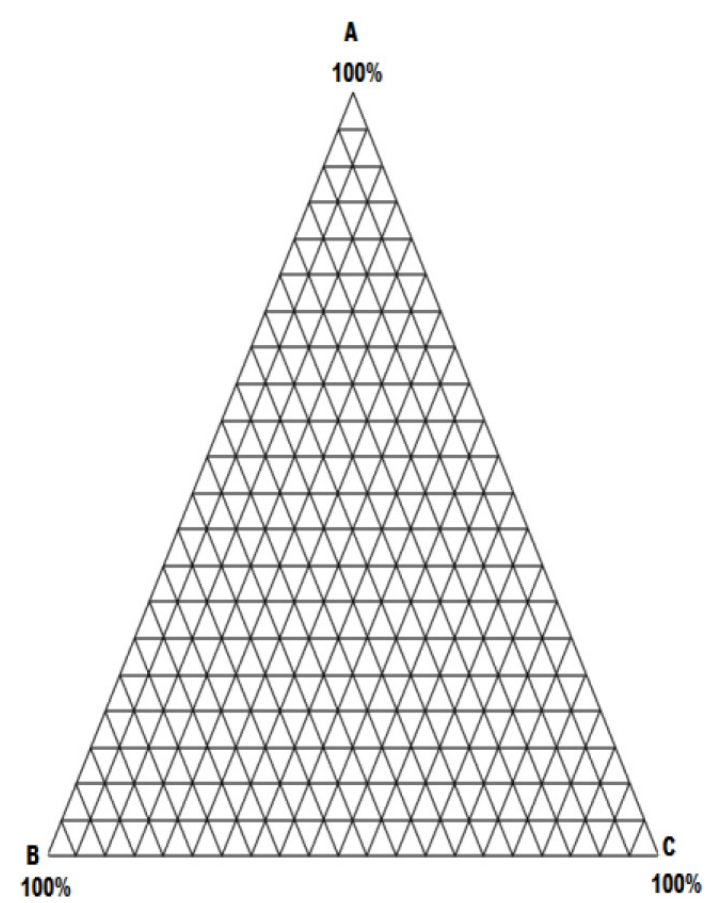

Figure 1 : Ternary Graph.

\section{PHASE DIAGRAMS FOR TERNARY MIXTURES}

It is also called as Gibbs phase triangle, triangle plot, ternary graph, simplex plot, Gibbs triangle or de Finetti diagram. A ternary system consists of three components. We can independently change the pressure, the temperature, and two independent composition variables for the system as a whole. ${ }^{8,13}$

A ternary or three component phase diagram has the shape of a triangular prism with an equilateral triangle as a foundation which is also called as composition triangle. In Figure 1, each apex of the triangle indicates one of the pure components A, B, or C. A point present on the edge of the triangle opposite a vertex represents a two component system or binary system and a point present within the internal area of triangle represents a three component system or ternary system. In a ternary system we need to limit the quantities of two components. So we call for two axes. The measure of the third can be obtained by deducting the aggregate of the two from 100 which means all three components $\mathrm{A}, \mathrm{B}$ and $\mathrm{C}$ represent $100 \%$ or sum of three is 100 . The entire space is divided into a set of small equilateral triangles which can be further subdivided. Smaller divisions give accurate and precise location of the composition. ${ }^{7,13}$

\section{METHODS OF READING AND RULES RELATING TO TRIANGULAR DIAGRAMS}

There are three usual methods which are applied to study a ternary phase diagram. The foremost method is an approximation based upon the 'phase diagram grid'. The triangle is divided into fine grids from accurate measurements of the components. The second method is the 'altitude method' (Shown in Figure 2a) which is used when the phase diagrams does not possess grid lines in which to determine the composition of all three components. The height or altitude of the triangle is set to $100 \%$ and the shortest distances is determined from the point of interest to each of the three sides or edges of the triangle. The content of each of the component can be estimated based on the distance calculated. The third method is 'intersection method' (Shown in Figure 2b) which is grounded upon a bigger number of measurements. It does not call for the drawing
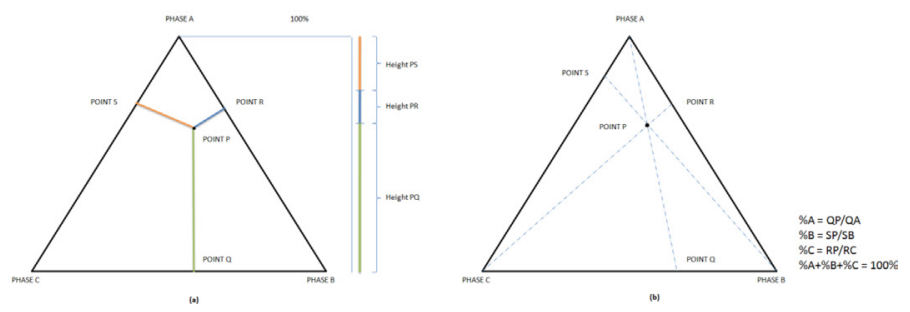

Figure 2 : (a) Altitude method (b) Intersection method, Modified from Ref. [8].

of perpendicular lines rather straight lines are drawn from each vertex or corner, to the opposite side of the triangle passing through the point of interest. The lengths of these lines, and the lengths of the segments between the point and the corresponding sides, are measured individually and the ratios of components $\mathrm{A}, \mathrm{B}$ and $\mathrm{C}$ can then be determined by dividing these segments by the entire corresponding line. ${ }^{8,13,14}$

It is very important to understand certain rules associated with the use of triangular coordinates. The concentration of the components in ternary systems is expressed in terms of weight basis.

Following statements should be studied: ${ }^{14}$

1. In Figure 3, each of the apexes of the equilateral triangle represents the $100 \%$ of one component in terms of weight and $0 \%$ of the remaining two components.

2. The three lines $(\mathrm{AB}, \mathrm{BC}, \mathrm{CA})$ joining the vertex points represent the combination of $\mathrm{A}, \mathrm{B}$ and $\mathrm{C}$, they represent the two component or binary mixtures. Now by dividing each line with 100 equal segments or units, the location of the point present along the line can be directly related to the $\%$ concentration of one component in a binary system.

3. In going along the edge of the triangle in order to represent the concentrations in a binary system. It does not matter whether we go in clockwise or anticlockwise direction, provided we are constant. For example in case of side $A B$ if we move in the direction of $B$, it signifies a binary system of $\mathrm{A}$ and $\mathrm{B}$ having the increasing concentrations of $\mathrm{B}$ and correspondingly decreasing concentration of A, similarly for BC and CA.

The region inside the triangle symbolises the all possible combinations of $\mathrm{A}, \mathrm{B}$ and $\mathrm{C}$ to give three component or ternary mixture.

For example, to determine the composition of $\mathrm{A}, \mathrm{B}$ and $\mathrm{C}$ at location ' $x$ ' in the triangle;

The side of the triangle $\mathrm{AC}$ opposite to apex $\mathrm{B}$ represents a binary systems composed of $\mathrm{A}$ and $\mathrm{C}$ in which concentration of component $\mathrm{B}$ is zero. The lines drawn parallel to side $\mathrm{AC}$ represents increasing concentration of B from 0 to $100 \%$ and the line that cut ' $\mathrm{x}$ ' is equivalent to $15 \%$ of $\mathrm{B}$ and $80 \%$ of $\mathrm{A}+\mathrm{C}$. Similarly along the line $\mathrm{AB} C=0$. The lines drawn parallel to $\mathrm{AB}$ shows increasing concentration of $\mathrm{C}$ from 0 to $100 \%$. The line parallel to $\mathrm{AB}$ that cut ' $\mathrm{x}$ ' is equivalent to $30 \%$ of $\mathrm{C}$. Thus the concentration of $\mathrm{A}$ can be calculated as $100-(B+C)=100-(15+30)=55 \%$.

5. Suppose a line is pulled through any corner of the triangle to a point on the opposite edge then all the systems present on the given line will have the same ratio of composition of the two components (ex in this case A and B). Further inclusion of $\mathrm{C}$ to a mixture of $\mathrm{A}$ and $B$ will give systems that lie progressively nearer to apex $C(100 \%$ of C)

6. Any line which is parallel to any side of the triangle (in Figure 3, Ex HI) represents the ternary systems in which proportion of any one component is constant. In this case $\mathrm{C}$ is constant and varying composition of $\mathrm{A}$ and $\mathrm{B}$. 


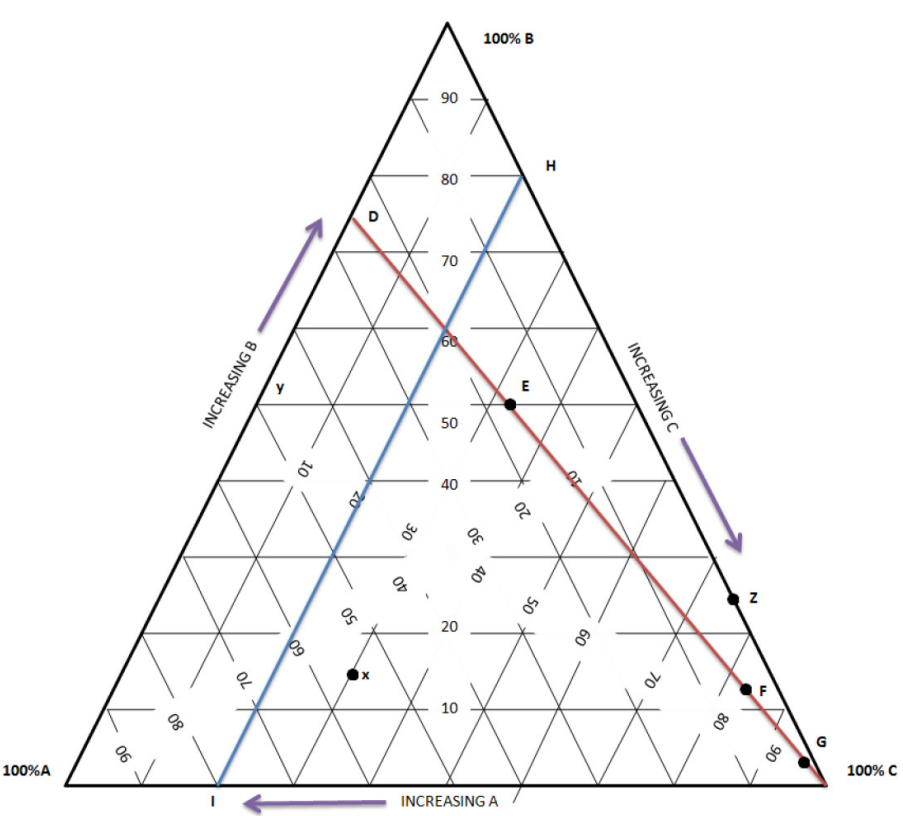

Figure 3 : Phase diagram for three component system, Modified from Ref. [14].

\section{THREE COMPONENT SYSTEM HAVING A PAIR OF MISCIBLE LIQUID}

Benzene and water are slightly miscible and they produce a binary system. Alcohol is completely miscible with both the solvents. Thus, it is expected that additions of sufficient alcohol to a two phase system would give a single phase system composed all the three components. Addition of alcohol leads to complete miscibility of two solvents which is achieved by solvent effect. Water is highly polar whereas benzene is non polar, alcohol is as an intermediate polar solvent that provides the electronic equilibrium and provides solvation. ${ }^{16}$

Suppose in Figure 4, A, B and C are three corners of a composition triangle representing water, alcohol and water respectively. The line $\mathrm{AC}$ represents a binary mixture of $\mathrm{A}$ and $\mathrm{C}$. The limits of solubility of $\mathrm{C}$ in $\mathrm{A}$ and $\mathrm{A}$ in $\mathrm{C}$ is denoted by $a$ and $c$ respectively at the specific temperature being used. The curve afdeic marks the extent of two phase region and the remaining triangle contains single phase liquid. The direction of tie line within the curve depends on the relative solubility of the third component on the other two components. The systems $g$ and $h$ present on the tie line $f i$ gives rise to two phases having composition denoted by points $f$ and $i$. The amount of two conjugate phases will depend on the position of the original system on the tie line. For example point $g$ after getting equilibrium and will separate into two phases $\mathrm{f}$ and $\mathrm{i}$ and its ratio on weight basis is given by gi: fi. Similarly for mixture $h{ }^{14}$

The incorporation of component B to a 50:50 blend of components A and $C$ produces a single phase system at point $d$. Similarly a 25:75 mixture of $\mathrm{A}$ and $\mathrm{C}$ is shown at point $j$ and addition of $\mathrm{B}$ leads to phase change at point $e .^{14}$

\section{WINSOR PHASE BEHAVIOUR DIAGRAMS}

Winsor on the basis of his study classified the water-oil-surfactant ternary mixture in four different phase equilibria (Shown in Figure 5). ${ }^{10}$

Type I: Water phase rich in surfactant also called as lower phase coexists with surfactant deficient oil phase. It is Winsor I type.

Type II: Oil phase rich in surfactant also called as the upper phase coexists with surfactant deficient water phase. It is Winsor II type.

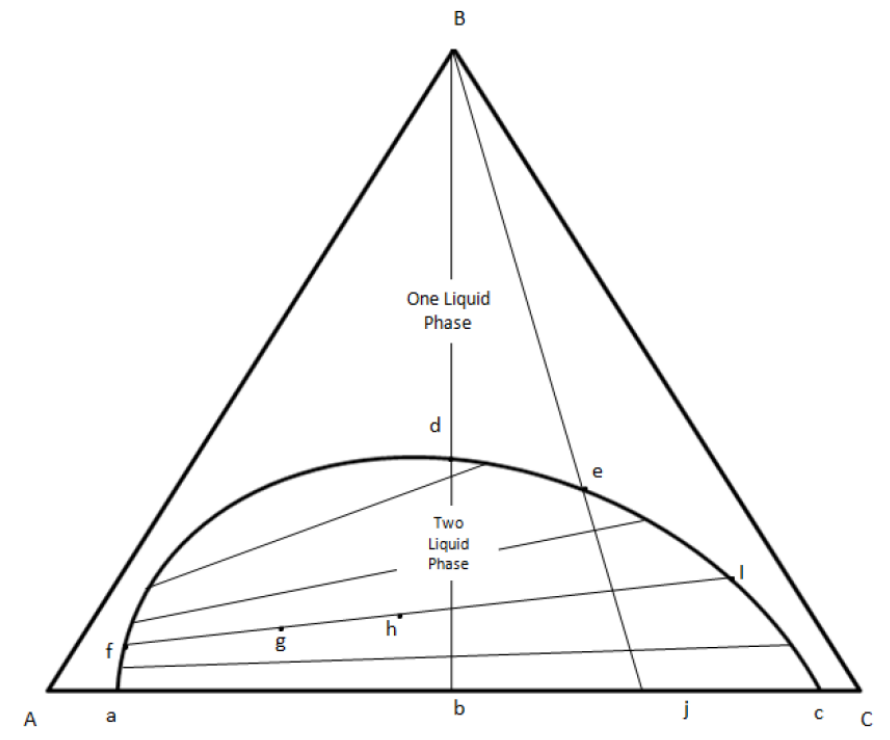

Figure 4 : Three component system, one pair of which is partially miscible, Modified from Ref. [14].

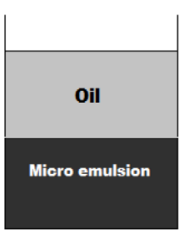

Winsor I

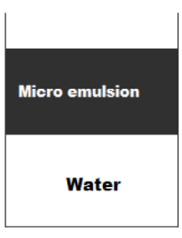

Winsor II

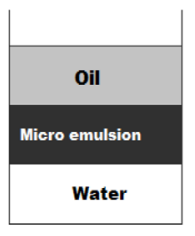

Winsor III

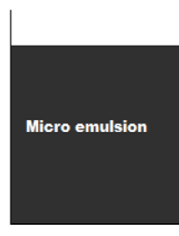

Winsor IV
Figure 5 : Winsor phase behaviour diagram, Modified from Ref. [10].

Type III: Surfactant rich middle phase coexists with both water (which is lower phase) and oil (which is upper phase) surfactant deficient phases. It is Winsor III type.

Type IV: Single phase homogeneous mixture. It is Winsor IV type.

Suppose, in Figure 6 a triangle is obtained by combining three components (Water-oil-Surfactant). It contains a one phase region which is called as region of miscibility and a two phase region which is called as region of immiscibility. The one phase contains the water in oil or oil in water micelles and it is homogeneous in micrometer or in optical range. The two phases contains a phase of water and surfactant and other is mostly oil at low temperature. A change from 1 phase to 2 phases promotes phase separation that promotes along tie lines. The critical condition for ternary mixture is called is plait point which is equivalent to critical point in binary mixture and the line is called as plait line. As the system point reaches the plait point or critical point from within the two-phase area, the length of the tie line through the system point approaches zero, the miscibility gap disappears, and the composition of the two liquid phases become indistinguishable. ${ }^{11,12}$

This is valid in low temperature. As the temperature increases it reaches the closed loop region which added two 2 phase regions to the phase diagram (one oil rich and other water rich) and two plait or critical points. Slop of the tie lines also changes. Such phase diagram is characterised by 3 phase regions in triangle inside the triangle which cuts two 2 phase regions. The cause of formation of 3 phase region is the temperature variable. ${ }^{11,12}$ 


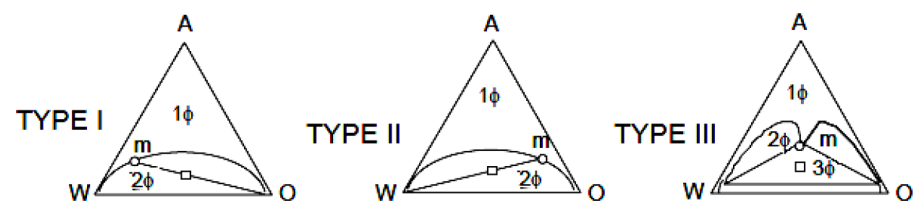

Figure 6 : Winsor's three ternary phase diagram types, Modified from Ref. [12].

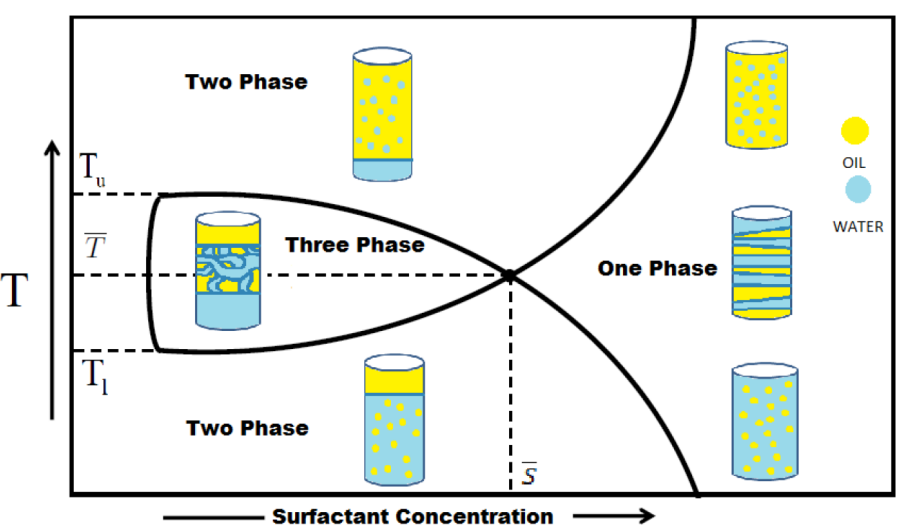

Figure 7 : The fish diagram, Modified from Ref. [9].

\section{Effect of Temperature}

In case of non-ionic micro emulsion, phase behaviour is temperature dependent. There is specific phase inversion temperature (PIT). As the temperature changes, the oil or water phase volume or the relative amount changes. At low Temperature or when $\mathrm{T}<\mathrm{PIT}$, the two phase consists of an oil phase and water-surfactant phase which is Winsor I. With the increase in the temperature till $\mathrm{T}=\mathrm{PIT}$, surfactant gets equally distributed between a water and an oil phase which is Winsor III type. Further increase in temperature or when T $>$ PIT, the two phase mixture becomes a water phase and an oil-surfactant phase which is Winsor II type. ${ }^{4,9,11}$

\section{THE FISH DIAGRAM}

The fish phase diagram can be obtained by taking a section across the ternary phase diagram along the isopleth line (Shown in Figure 7). This cut correlates with change in the temperature and composition of one component but the ratio of remaining two components remains fixed. Thus a section through Gibbs phase triangle at constant water to oil ratio and varying surfactant concentration is called as fish cut phase diagram. The name implies characteristic fish like shape.,

The fish head shows the three phase region whereas fish tail shows one phase region. The three phase region extends from $T_{1}$ to $T_{u}$ where $T_{u}$ and $\mathrm{T}_{1}$ are highest and lowest temperatures where three phase region exist. The temperature at which fish body intersects fish tail is known as phase inversion temperature (PIT) or also called as tricritical point which is dependent on the components of the emulsion and surfactant concentration. Phase inversion temperature or tricritical temperature represents the efficacy of surfactant or the minimum amount of surfactant required for complete emulsification of oil and water. Above $T_{u}$ and below $T_{1}$ it is two phase region. ${ }^{9,11}$

\section{CONSTRUCTION OF A PHASE DIAGRAM}

Sheikh Shafiq-un-Nabi et al. have worked on development of nanoemulsion and its optimization. They constructed ternary phase diagram using aqueous titration method for their study. Different ratios of surfactant and cosurfactant (Smix) in different volume were used to study the phase diagrams in nanoemulsion formation. For each phase diagram specific Smix ratio was thoroughly mixed in different volumes of oil. Aqueous titration method was used for the development of the ternary phase diagram in which each oil and Smix combination was slowly titrated with aqueous phase. The amount of water added was calculated in the range of $5 \%$ to $95 \%$ of total volume, with the increment of $5 \%$ after each

\begin{tabular}{|c|c|c|c|c|c|c|c|c|}
\hline \multicolumn{9}{|c|}{ Percentage of Oil, Smix and water used in the construction of phase diagram. (Oil and Smix is in the ratio of 1:9) } \\
\hline Oil(mg) & Smix (mg) & Water(mg) & Water added & Total(mg) & Oil (\%) & Smix (\%) & Water (\%) & \\
\hline 20 & 180 & 20 & 0 & 220 & 9.09 & 81.82 & 9.09 & \\
\hline 20 & 180 & 40 & 20 & 240 & 8.33 & 75 & 16.67 & \\
\hline 20 & 180 & 50 & 10 & 250 & 8 & 72 & 20 & \\
\hline 20 & 180 & 70 & 20 & 270 & 7.41 & 66.67 & 25.93 & \\
\hline 20 & 180 & 90 & 20 & 290 & 6.9 & 62.07 & 31.03 & \\
\hline 20 & 180 & 110 & 20 & 310 & 6.45 & 58.06 & 35.48 & 01:09 \\
\hline 20 & 180 & 130 & 20 & 330 & 6.06 & 54.55 & 39.39 & \\
\hline 20 & 180 & 160 & 30 & 360 & 5.56 & 50 & 44.44 & \\
\hline 20 & 180 & 200 & 40 & 400 & 5 & 45 & 50 & \\
\hline 20 & 180 & 240 & 40 & 440 & 4.55 & 40.91 & 54.55 & \\
\hline 20 & 180 & 300 & 60 & 500 & 4 & 36 & 60 & \\
\hline 20 & 180 & 370 & 70 & 570 & 3.51 & 31.58 & 64.91 & \\
\hline 20 & 180 & 470 & 100 & 670 & 2.99 & 26.87 & 70.15 & \\
\hline 20 & 180 & 600 & 130 & 800 & 3 & 23 & 75 & \\
\hline 20 & 180 & 800 & 200 & 1000 & 2 & 18 & 80 & \\
\hline 20 & 180 & 1100 & 300 & 1300 & 2 & 14 & 85 & \\
\hline 20 & 180 & 1800 & 700 & 2000 & 1 & 9 & 90 & \\
\hline 20 & 180 & 4000 & 2200 & 4200 & 0.48 & 4.29 & 95.24 & \\
\hline
\end{tabular}


addition and was mixed in vortex mixer. Based on the Visual observation the following categories were assigned:

1. Transparent and easily flowable: Nanoemulsion (N).

2. Transparent gel: Nanogel (NG).

3. Milky/cloudy: Emulsion (E).

4. Milky gel: Emulgel (EG).

The obtained observations were tabled and percentage of oil, surfactant and cosurfactant were calculated. Only nanoemulsion points were plotted as the nanoemulsion area is of interest. ${ }^{16}$

\section{Example}

For an example of construction of ternary phase diagrams, in formulation of self emulsifying dosage form is explained below. Different Smix ratios (surfactant and co surfactants) of 4:1, 3:1, 2:1, 1:1, 1:2, 1:3, 1:4 were used along with oily phase to construct different phase diagrams. Water titration method was used for the phase diagram construction and visual inspection was performed. Observed phases after each addition were tabulated. Following phase diagram percentage table was made for each Oil to Smix ratios (1:9 to 1:1). Phase diagram was constructed suing CHEMIX School v.3.51 software.

Similar tables were made for each ratio of oil and Smix. Phase diagrams were constructed to determine optimum concentration of oil, surfactant, and co-surfactant. Larger the size of nano emulsion region in ternary phase diagram, greater is the self-emulsification efficiency. Smix 3:1 ratio showed a larger nano emulsion region and hence selected.

\section{RECENT APPLICATIONS OF TERNARY PHASE DIAGRAMS IN PHARMACEUTICALS}

Figure 8 illustrated various application of ternary phase diagram in pharmaceutical.

1. Muhammad Naeem et al. have Worked on development and optimization of Lornoxicam microemulsion formulation and used ternary phase diagram for selection of components of microemulsion and their ranges also the selection of microemulsion area. ${ }^{15}$

2. Sheikh Shafiq-un-Nabi et al. have worked on development of nanoemulsion and its optimization. They constructed ternary phase diagram using aqueous titration method for their study. ${ }^{16}$

3. Haroon k. Syed et al. used ternary phase diagram to identify and select the optimal blends of oil and surfactant and study their phase behaviour. It also helped them to determine different type of dispersions formed at different concentrations of components. ${ }^{17}$

4. Peter J. Dowding et al. have worked on Oil core containing polymer shell microcapsules they used ternary diagrams to study the phase behaviours of ternary mixture of polystyrene, hexadecane and dichloromethane and determine the point of phase separation which gives the region where the solid polymer shell forms. ${ }^{18}$

5. Hayder Kadhim Drais et al. have worked on nanoemulsion of carvidelol as oral liquid dosage form. They made ternary phase diagrams using aqueous titration method to get the region of better nano emulsifying activity. ${ }^{19}$

6. Hany M. El-Banna have worked on solid dispersions and used the ternary phase diagram of Aspirin, Acetaminophen and Urea System in his studies. ${ }^{20}$

7. S.K. Mehta et al have worked on tween based rifampicin microemulsion for which they used ternary plot to determine the microemulsion region of Tween80, ethanol, oleic acid, Phosphate buffer ph $(7.4)$ at $30^{\circ} \mathrm{C}$ and found that the ternary phase diagram and microemulsion region for Tween 80 -ethanol-oleic acid-phosphate buffer was delineated. ${ }^{21}$

\section{Smix ratio 3:1}

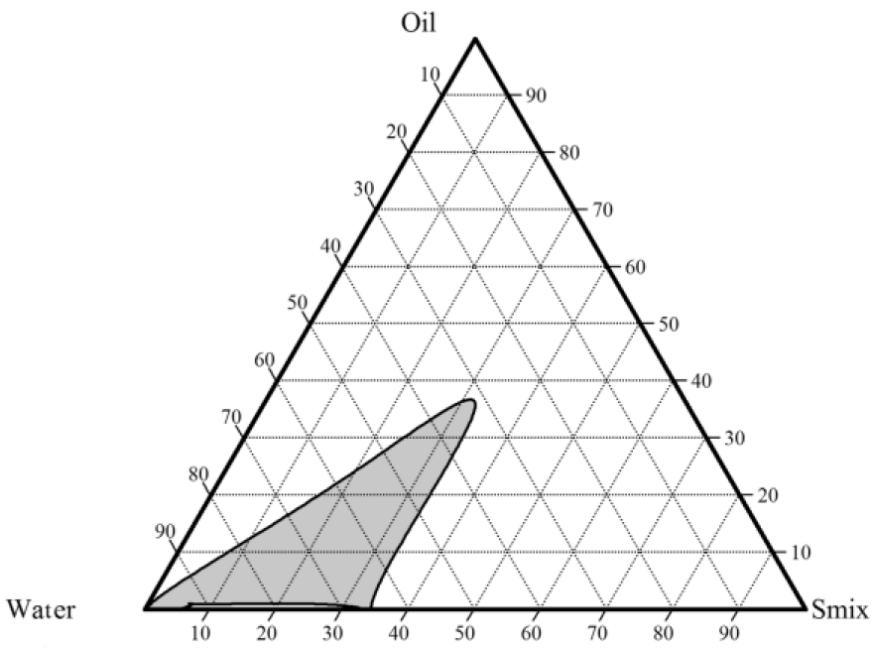

Figure 8: Phase diagram for 3:1 Smix ratio.

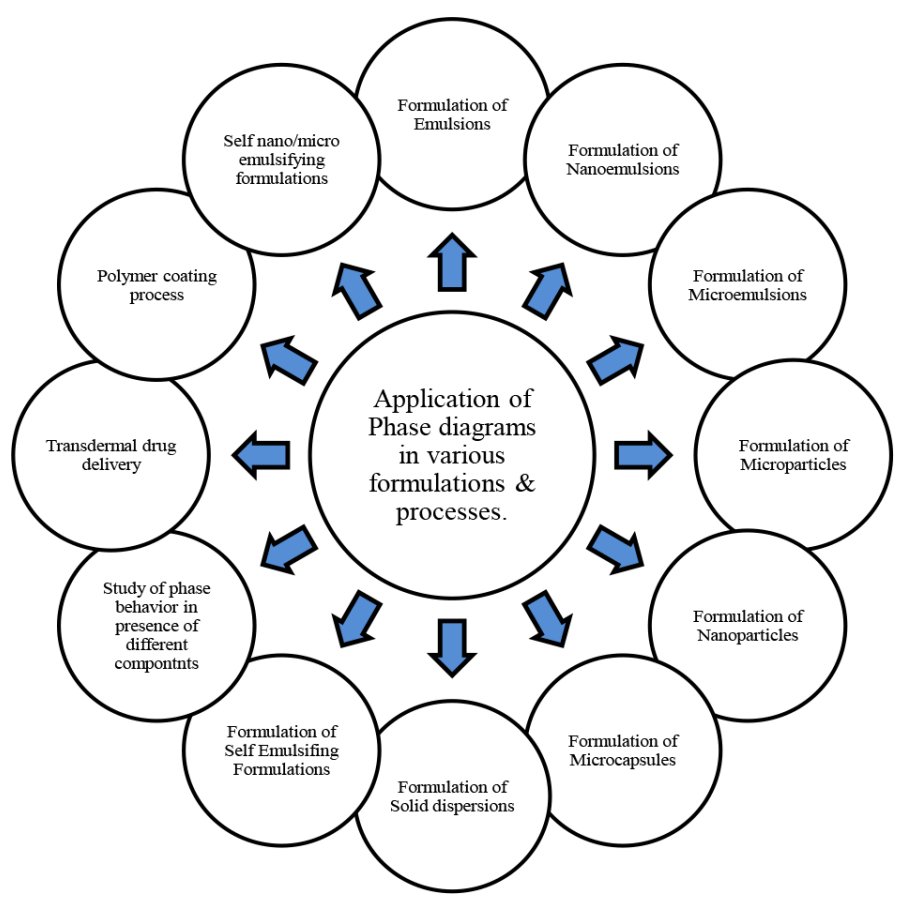

Figure 9 : Various applications of ternary phase diagrams. ${ }^{15-29}$

8. Yulu Wang et al. worked on polymer coating of nanoparticles using supercritical antisolvent method for which they used ternary phase diagram. ${ }^{22}$

9. Lanlan Wei et al. have worked on self emulsifying drug delivery system (SEDDS) of drug Carvedilol for which they examined ternary phase diagram to determine the existence of self emulsifying fields. ${ }^{23}$

10. Anna Czajkowska-Ko'snik et al. have worked on liquid and solid self-emulsifying drug delivery systems for Atorvastatin and used ternary phase diagram in their study. ${ }^{24}$

11. Peter p. Guerrieri et al. have worked on phase behaviour of Ranitidine hydrochloride in the presence of degradants and atmospheric 
moistures and its impact on chemical stability and used ternary phase diagram to visualize the proportion of the drug in the solid and solution phases as a function of impurity and moisture content. ${ }^{25}$

12. Rajesh Kumar et al. have worked on Telmisartan nanoemulsion and they used ternary phase diagram for screening of excipients and determination of range of nanoemulsion by using aqueous titration method. ${ }^{26}$

13. Jaehwi Lee et al. have worked on Aceclofenac microemulsion for transdermal delivery and used ternary phase diagram to obtain the concentration range of oil, surfactant, Cremophor ELP, and co-surfactant, ethanol for microemulsion formation. ${ }^{27}$

14. Kausar Shafaat et al. have worked on transdermal delivery of Clozapine using nanoemulsion as vehicles and used ternary phase diagram to determine extent and nature nanoemulsion region and to select different formulations. ${ }^{28}$

15. Yotsanan Weerapol et al. have worked on emulsification of Nifedipine self-nano emulsifying drug delivery system (SNEDDS) and used ternary phase diagram to determine the region giving SNEDDS and comparison of every ratio of selected surfactant and oil can be to select the surfactant, co-surfactant, and oil combinations. ${ }^{29}$

\section{CONCLUSION}

Ternary phase diagram construction is the best approach to study all the types of formulations that can be formulated by mixing of water, oil and surfactant. It provides the scientific basis for the screening of different formulation components and determination of optimum conditions for existence of equilibrium. It is widely used in the research and formulation studies of various pharmaceutical formulations and processes.

\section{ACKNOWLEDGEMENT}

The author would like to thank Manipal College of Pharmaceutical Science, Manipal Academy of Higher Education for providing the support.

\section{CONFLICT OF INTEREST}

The Author reports no conflict of interest regarding the publication of this paper.

\section{ABBREVIATIONS}

PIT: phase inversion temperature; SEDDS: Self emulsifying drug delivery system.

\section{REFERENCES}

1. West $D$, Saunders N. Ternary phase diagrams in materials science. $3^{\text {rd }}$ ed. CRC press. 2017.

2. Singh D, Deshwal B, Vats S. Comprehensive engineering chemistry. New Delhi: I K International Publishing House. 2007.

3. Atkins P, De Paula J. Atkins' Physical chemistry. $8^{\text {th }}$ ed. Oxford: Oxford University Press. 2006

4. Microemulsion [Internet]. Http://www.chm.bris.ac.uk. 2004 [cited 23 July 2017]. Available from: http://www.chm.bris.ac.uk/eastoe/Surf_Chem/3\%20Microemulsions.pdf

5. Physical Pharmacy [Internet]. Http://qu.edu.iq/. [Cited 18 July 2017]. Available from: http://qu.edu.iq/ph/wp-content/uploads/2015/03/lec-6.pdf

6. Bahl B, Bhal A, Tuli G. Essentials of physical chemistry. New Delhi: S. Chand \&
Company Ltd. 2008.

7. Module 25 Ternary Phase Diagram [Internet]. Nptel ac in. 2015. [cited 18 July 2017]. Available from: http://nptel.ac.in/courses/113105023/Lecture25.pdf

8. Vaughan, Will (September 5, 2010). "Ternary plots". Retrieved September 7. 2010.

9. Paroor H. Microemulsion: Prediction of the Phase diagram with a modied Helfrich free energy Ha. Max Planck Institute for Polymer Research Mainz Germany and Johannes Gutenberg University, Mainz, Germany. 2012.

10. Guleri T, Kaur L, Tarun G, Srivastava D, Thakur S. Micro emulsion: a promising approach for controlled drug release. Int J Appl Biol Pharm (IRJPAS). 2013; $3(1): 102-8$.

11. Chapter 42 - phase diagrams for micellar systems [Internet]. https://www.ncnr. nist.gov. [Cited 19 July 2017]. Available from: https://www.ncnr.nist.gov/staff/ hammouda/summer_school/chapter_42.pdf

12. Nielloud F, Marti-Mestres G. Pharmaceutical emulsions and suspensions. New York: Marcel Dekker, Inc. 2000

13. 3 Phase Diagrams: Ternary Systems [Internet]. Chemistry LibreTexts 2015. [cited 18 July 2017]. Available from: https://chem.libretexts.org/ Textbook_Maps/Physical_and_Theoretical_Chemistry_Textbook_Maps/ DeVoe's_\%22Thermodynamics_and_Chemistry\%22/13\%3A_The_Phase_ Rule_and_Phase_Diagrams/13.3_Phase_Diagrams\%3A_Ternary_Systems

14. Martin A, Sinko P, Singh Y. Martin's physical pharmacy and pharmaceutical sciences. 6th ed. Wolter Kluwer/Lippincott Williams and Wilkins. 2011.

15. Naeem M, Rahman N, Khan J, Sehti A, Nawaz Z. Development and Optimization of Microemulsion Formulation using Box-Behnken Design for Enhanced Transdermal Delivery of Lornoxicam. Latin American Journal of Pharmacy. 2013;32(8):1196-204.

16. Shafiq-un-Nabi S, Shakeel F, Talegaonkar S, Ali J, Baboota S, Ahuja A et al. Formulation Development and Optimization Using Nanoemulsion Technique: A Technical Note. AAPS Pharm Sci Tech. 2007;8(2):E1-E5.

17. Syed H, Peh K. Identification of phases of various oil, surfactant/ co-surfactants and water system by ternary phase diagram. Acta poloniae pharmaceutica $\tilde{n}$ drug Research. 2014;71(2):301-9.

18. Dowding P, Atkin R, Vincent B, Bouillot P. Oil Core-Polymer Shell Microcapsules Prepared by Internal Phase Separation from Emulsion Droplets. I. Characterization and Release Rates for Microcapsules with Polystyrene Shells. Langmuir. 2004;20(26):11374-9.

19. Drais $H$, Hussein A. Formulation and characterization of carvedilol nanoemulsion oral liquid dosage form. International Journal of Pharmacy and Pharmaceutical Sciences. 2015;7(12):211-6.

20. Solid Dispersion of Pharmaceutical Ternary Systems I: Phase Diagram of Aspirin-Acetaminophen-Urea System. Journal of pharmaceutical Sciences. 1978;67(8):1109-11.

21. Mehta S, Kaur G, Bhasin K. Analysis of Tween based microemulsion in the presence of TB drug rifampicin. Colloids and Surfaces B: Biointerfaces. 2007;60(1):95-104.

22. Wang Y, Dave R, Pfeffer R. Polymer coating/encapsulation of nanoparticles using a supercritical anti-solvent process. J Supercrit Fluids. 2004;28(1):85-99.

23. Wei L, Sun P, Nie S, Pan W. Preparation and Evaluation of SEDDS and SMEDDS Containing Carvedilol. Drug Development and Industrial Pharmacy. 2005;31(8):785-94.

24. Czajkowska-Kosnik A, Szekalska M, Amelian A, Szymanska E, Winnicka K Development and Evaluation of Liquid and Solid Self-Emulsifying Drug Delivery Systems for Atorvastatin. Molecules. 2015;20(12):21010-22.

25. Guerrieri P, Smith D, Taylor L. Phase Behavior of Ranitidine $\mathrm{HCl}$ in the Presence of Degradants and Atmospheric Moistures Impact on Chemical Stability. Langmuir. 2008;24(8):3850-6.

26. Kumar R, Soni G, Prajapati S. Formulation development and evaluation of Telmisartan Nanoemulsion. Int J Res Dev Pharm I Sci. 2017;6(4):2711-9.

27. Lee J, Lee $Y$, Kim J, Yoon M, Choi Y. Formulation of microemulsion systems for transdermal delivery of aceclofenac. Archives of Pharmacal Research. 2005;28(9):1097-2.

28. Shafaat K, Kumar B, Das S, Hasan R, Prajapati S. Novel nanoemulsion as vehicles for transdermal delivery of clozapine: in vitro and in vivo studies. International Journal of Pharmacy and Pharmaceutical Sciences. 2013;5(3):126-34.

29. Weerapol Y, Limmatvapirat S, Kumpugdee-Vollrath M, Sriamornsak P. Spontaneous Emulsification of Nifedipine-Loaded Self-Nanoemulsifying Drug Delivery System. AAPS Pharm Sci Tech. 2015;16(2):435-43. 